\title{
Fluorescein angiography and optical coherence tomography in myopic choroidal neovascularization
}

J Chhablani', MJ Deepa'1, M Tyagi'1'R Narayanan and I Kozak ${ }^{2}$

\begin{abstract}
Purpose To assess intra/inter-observer agreement, and diagnostic capabilities of a color fundus photograph, fundus fluorescein angiography (FFA), and spectral domain optical coherence tomography (SD-OCT) in making a diagnosis of myopic choroidal neovascularization (CNV).

Patients and methods Two masked observers evaluated FFA and SD-OCT images to identify the presence of myopic CNV in 80 high-myopic eyes of 57 patients. A third masked observer identified CNV on a color fundus photo. Presence of myopic $\mathrm{CNV}$ on a fundus photo was defined as presence of subretinal hemorrhage, thickening of the retina and/or visible membrane at the macula. Presence of myopic CNV on FFA was defined as hyperfluorescence in the early phase with increase in intensity and size in the late phase; presence of a large irregular lesion; and hypofluorsescence due to subretinal hemorrhage. Myopic CNV on SD-OCT was defined as the hyper-reflective lesion with or without intraretinal fluid or subretinal fluid with retinal thickening. Results Intraobserver repeatability on FFA and SD-OCT was 0.54 and 0.44 , respectively. Agreement (kappa) between FFA and SD-OCT was 0.38 and 0.3 , respectively. Among 34 eyes, which had the presence of $\mathrm{CNV}$ on a color fundus photo, $\mathrm{CNV}$ was diagnosed in $18(53 \%)$ eyes on FFA and in $20(58.8 \%)$ eyes on SD-OCT. Sensitivity and specificity of FFA was 47 and $80.4 \%$, respectively, and that of SD-OCT was 58.8 and $86.9 \%$, respectively.

Conclusion Repeatability and reproducibility for diagnosis of myopic $\mathrm{CNV}$ was better with FFA compared with SD-OCT;
\end{abstract}

however, agreement is very poor between FFA and SD-OCT. SD-OCT is comparatively a better tool to rule out presence of myopic CNV.

Eye (2015) 29, 519-524; doi:10.1038/eye.2014.345; published online 23 January 2015

\section{Introduction}

High myopia is one of the major causes of blindness throughout the world. ${ }^{1,2}$ In general population, high myopia has a prevalence of $1-2 \%,{ }^{1}$ however, it affects $6-27 \%$ of all myopic eyes. ${ }^{3}$ Incidence of myopia in the south Indian population (36.5\%) was found to be much higher when compared with the west. ${ }^{4}$ High myopia is defined by an axial length of the eye higher than $26 \mathrm{~mm}$ or by a refractive error of more than -6 diopters (D). ${ }^{5}$ Choroidal neovascularization (CNV) growing between the retinal pigment epithelium and neurosensory retina (type $2 \mathrm{CNV}$ ) is the most common central vision-threatening complication in patients with high myopia. Ohno-Matsui $e t a l^{6}$ reported the incidence of myopic CNV as $10.2 \%$. Myopic $\mathrm{CNV}$ may account for as much as $62 \%$ of $\mathrm{CNV}$ occurring in patients younger than 50 years of age. ${ }^{7}$

$\mathrm{CNV}$ associated with high myopia could be difficult to diagnose due to associated chorioretinal atrophy, pigmentary changes, visibility of large choroidal vessels, and scarring. As these patients have poor visual acuity due to posterior staphyloma or chorioretinal atrophy, sometimes, the presence of CNV in such eyes could be an incidental finding. Patients with large chorioretinal atrophy at the posterior pole may have good vision due to a small area of normal retinal
${ }^{1}$ Srimati Kanuri Santhamma Vitreo-Retina Service, L.V. Prasad Eye Institute, Hyderabad, India

${ }^{2}$ Division of Vitreoretinal Diseases and Surgery, King Khaled Eye Specialist Hospital, Riyadh, Kingdom of Saudi Arabia

Correspondence:

J Chhablani, Srimati Kanuri Santhamma Vitreo-Retina Service, L.V. Prasad Eye Insitute, L.V. Prasad Marg, Banjara Hills, Hyderabad, AP 500034, India Tel: +9140 30612607; Fax: +914023548271 . E-mail: jay.chhablani@ gmail.com

Received: 1 March 2014 Accepted in revised form: 23 October 2014 Published online: 23 January 2015

This work has previously been presented at the All India Ophthalmic Society Conference, Agra, 2014. 
pigment epithelium. Sudden vision loss in such patients could be due to myopic CNV. However, clinical examination may not be able to reveal any obvious myopic CNV due to large chorioretinal atrophy.

In a clinical setting, it is very difficult to make a diagnosis of $\mathrm{CNV}$ in a high myope who presents with sudden loss of vision. Making a correct diagnosis with the help of available diagnostic modalities, which are customized for emmetropic eyes, becomes a challenging task in order to avoid needless treatment. Therefore, we were interested to evaluate the correlation between presenting symptoms, color photograph, fundus fluorescein angiography (FFA), and spectral domain optical coherence tomography (SD-OCT) findings to understand the utility of each of this in making the diagnosis of myopic CNV.

The aim of this study was to assess the interobserver correlation in making a diagnosis of myopic CNV based on a color fundus photograph, FFA, and SD-OCT, and correlate it with presenting symptoms in subjects with suspected myopic CNV.

\section{Materials and methods}

In a retrospective study, 80 eyes from 57 patients with high myopia who presented to the LV Prasad Eye Institute, India from January 2008 to June 2012 were analyzed. Prior approval from the institutional review board was taken and informed consent was obtained from each subject. Inclusion criteria were eyes with high myopia that underwent FFA and SD-OCT for suspicion of presence of $\mathrm{CNV}$. Eyes with any other associated retinal pathology including diabetic retinopathy, drusen, or CNV secondary to age-related macular degeneration or any other cause, mild and moderate myopia (refractive error $<6 \mathrm{D}$ ), history of any prior vitreoretinal surgery, history of photodynamic therapy or intravitreal anti-VEGF (vascular endothelial growth factor) injections, laser, unavailability of any one imaging modality (color photograph, FFA, SD-OCT), and poorquality images were excluded.

Diagnostic criteria for high myopia was a refractive error of $-6 \mathrm{D}$ or more and various clinical features observed on fundus examination usually associated with high myopia, including posterior pole staphyloma, diffuse or patchy chorioretinal atrophy, peripapillary atrophy, tilted disc, lacquer cracks, and depigmentation of the fundus allowing good visualization of the choroidal vessels.

All participants underwent a comprehensive ocular examination including history of presenting complaints, best-corrected visual acuity testing, dilated fundus examination with slit lamp biomicroscopy, color fundus photography, digital FFA, and SD-OCT at baseline.

\section{Imaging}

Color photograph Color fundus photographs of the optic disc, macula, and temporal retina $\left(30^{\circ}\right)$ were captured with a mydriatic camera (Zeiss FF450, Carl Zeiss Meditec, Jena, Germany).

FFA All patients underwent a routine FFA procedure with intravenous injection of $5 \mathrm{ml}$ of $10 \%$ sodium fluorescein solution (Novartis Pharma AG, Bern, Switzerland). After the injection, images were taken from the early phase (10-20s) to the late phase ( $10 \mathrm{~min})$. A confocal scanning laser ophthalmoscope (HRA2; Heidelberg Engineering, Dossenheim, Germany) was used. From each series, one representative early-phase angiogram and one from the late phase were selected for analysis.

SD-OCT The SD-OCT scans were obtained using Cirrus HD-OCT (Carl Zeiss Meditec Inc., Dublin, CA, USA) after dilation of the pupil with $1 \%$ tropicamide and $10 \%$ phenylephrine eye drops. All patients were imaged using a macular cube $512 \times 128$ scan (128 B-scans $\times 512 \mathrm{~A}$-scans covering a retinal area of $6.0 \times 6.0 \mathrm{~mm}$ ) as well as HD 5-line raster scans.

All the imaging procedures were performed by certified optometrists.

\section{Image analysis}

Using a removable hard disc storage device, we exported all color fundus photos, FFA, and SD-OCT images in JPEG (Joint Photographic Experts Group) format and analyzed them on the same computer screen with the same resolution by both the masked observers.

Presence of myopic CNV on the color fundus photograph was defined as the presence of subretinal hemorrhage with associated thickening of the retina and/or visible membrane at the macula. Presence of myopic CNV on FFA was defined as hyperfluorescence in the early phase with increase in intensity and size in the late phase, presence of a membrane, large irregular lesion, and hypofluorsescence due to subretinal hemorrhage. Myopic CNV on SD-OCT was defined as the hyper-reflective lesion located above the retinal pigment epithelium-Bruch's membrane complex associated with or without intraretinal fluid (IRF) or subretinal fluid (SRF) and associated with the intraretinal hyper-reflective area with retinal thickening.

Two retinal specialists, as masked observers (JC and $\mathrm{RN}$ ) analyzed all the phases of FFA and all the scans of SD-OCT. One observer (JC) analyzed FFA and SD-OCT 
images twice at different time points and was masked to his previous observations. The third masked observer (DMJ) graded the color photographs. Observers graded the color photographs, FFA, and SD-OCT images separately to diagnose the presence of CNV secondary to high myopia. The grades were, grade 1: presence of CNV, grade 2: indeterminate, and grade 3: absence of CNV. All the masked observers adhered to the criteria mentioned above while grading the images. The details about the patients including presenting complaints were masked to all observers.

Correlation between the color photographs, FFA, and SD-OCT was performed. Statistical analyses were performed using microsoft excel and GraphPad Prism 5 (GraphPad Software Inc., La Jolla, CA, USA).

\section{Results}

Total 116 eyes of 68 subjects' charts and images were reviewed for the study. However, 36 eyes were excluded due to poor image quality of either FFA (21 eyes) or SD-OCT (15 eyes). Therefore, a total 80 eyes of 57 subjects were included. There were 32 males and 25 females. Median age was 50 years (range 16-85 years). Median refractive error was $-10 \mathrm{D}$ (range -6 to $-34 \mathrm{D}$ ).

There were no presenting complaints in 37 eyes (22 subjects). Presenting complaint was diminution of vision in 43 eyes (35 subjects) with a mean duration of vision loss of 42 days (range 1 day to 3.5 months).

Common fundus findings were pigmentary changes (10 eyes), peripapillary atrophy (70 eyes), subretinal hemorrhage (27 eyes), and a greyish membrane (35 eyes). Four eyes were pseudophakic and five eyes had a history of refractive surgery.

Two masked observers analyzed a total of 80 sets of images, both FFA and SD-OCT. Intraobserver repeatability for the observer (JC) making the diagnosis of myopic CNV on FFA and SD-OCT was 0.54 and 0.44 , respectively. Agreement (kappa) between FFA and SDOCT for making the diagnosis of myopic CNV was 0.38 and 0.3 , respectively.

Among the 80 eyes, CNV was identified on a color fundus photo in 34 eyes, as there was presence of subretinal hemorrhage and/or a greyish membrane. Out of 34 eyes, diagnosis of CNV was made on FFA and SD-OCT in 18 (53\%) and $20(58.8 \%)$ eyes, respectively. Diagnostic abilities of FFA and SD-OCT, considering the color fundus photo as a gold standard, are shown in Table 1.

Symptomatic eyes were analyzed separately to correlate patient's symptoms with clinical and diagnostic findings. Out of 43 symptomatic eyes, CNV on the color photo was present in $23(53.4 \%)$ eyes; CNV was diagnosed on FFA and SD-OCT in $22(51.1 \%)$ eyes and
Table 1 Diagnostic ability of fluorescein angiography (FFA) and spectral domain optical coherence tomography (SD-OCT) in eyes with myopic choroidal neovascularization on clinical examination

\begin{tabular}{llc}
\hline & FFA & SD-OCT \\
\hline Sensitivity (\%) & 47 & 58.8 \\
Specificity (\%) & 80.4 & 86.9 \\
Positive predictive value (\%) & 64 & 76.9 \\
Negative predictive value (\%) & 67 & 74 \\
\hline
\end{tabular}

$18(41.8 \%)$ eyes, respectively. Representative cases are shown as Figures 1 and 2.

\section{Discussion}

Our study evaluates imaging modalities in diagnosis of CNV in high-myopic eyes. Intraobserver correlation was better with the FFA (0.54) compared with SD-OCT (0.44). Similarly, correlation between two masked observers was slightly better with FFA (0.38) compared with SD-OCT (0.3). This supports that FFA has more consensus between the clinicians for making a diagnosis of myopic CNV. This is similar to the previously reported studies in cases of CNV secondary to age-related macular degeneration, as well as myopic CNV. ${ }^{8,9}$ Milani et al ${ }^{9}$ reported overall agreement of 0.53 between the observers for FFA in eyes with myopic CNV.

When FFA and SD-OCT were compared with each other, poor agreement of 0.37 was found. Leveziel et al ${ }^{10}$ also reported a poor agreement $(0.25)$ between FFA and SD-OCT. The probable reasons for this poor agreement could be the small size of myopic CNV, less exudative signs, and associated architectural change at the posterior pole in high myopes. ${ }^{11}$

Recently, Leveziel et $a l^{10}$ reported contribution of FFA and SD-OCT in diagnosis of myopic CNV. They included recently diagnosed cases of myopic $\mathrm{CNV}$, and found that reliable and interpretable diagnosis of CNV was made by FFA alone in $61.3 \%$ of cases $(38 / 62)$, by SD-OCT alone in $22.6 \%$ of cases $(14 / 62)$, and by both SD-OCT and FFA in $16.1 \%$ of cases $(10 / 62)$. However, the observers were not masked and probably biased, as all the cases were diagnosed as myopic CNV. Authors did not evaluate the intra/inter-observer reproducibility. As discussed previously, owing to challenges in making the diagnosis of myopic $\mathrm{CNV}$, these parameters are important. We also correlated patients' symptoms and analyzed images in masked fashion in unconfirmed cases of myopic CNV. Another study by Milani et $a l^{9}$ reported that masked observers could identify CNV in only $15.5 \%$ cases by FFA alone. 

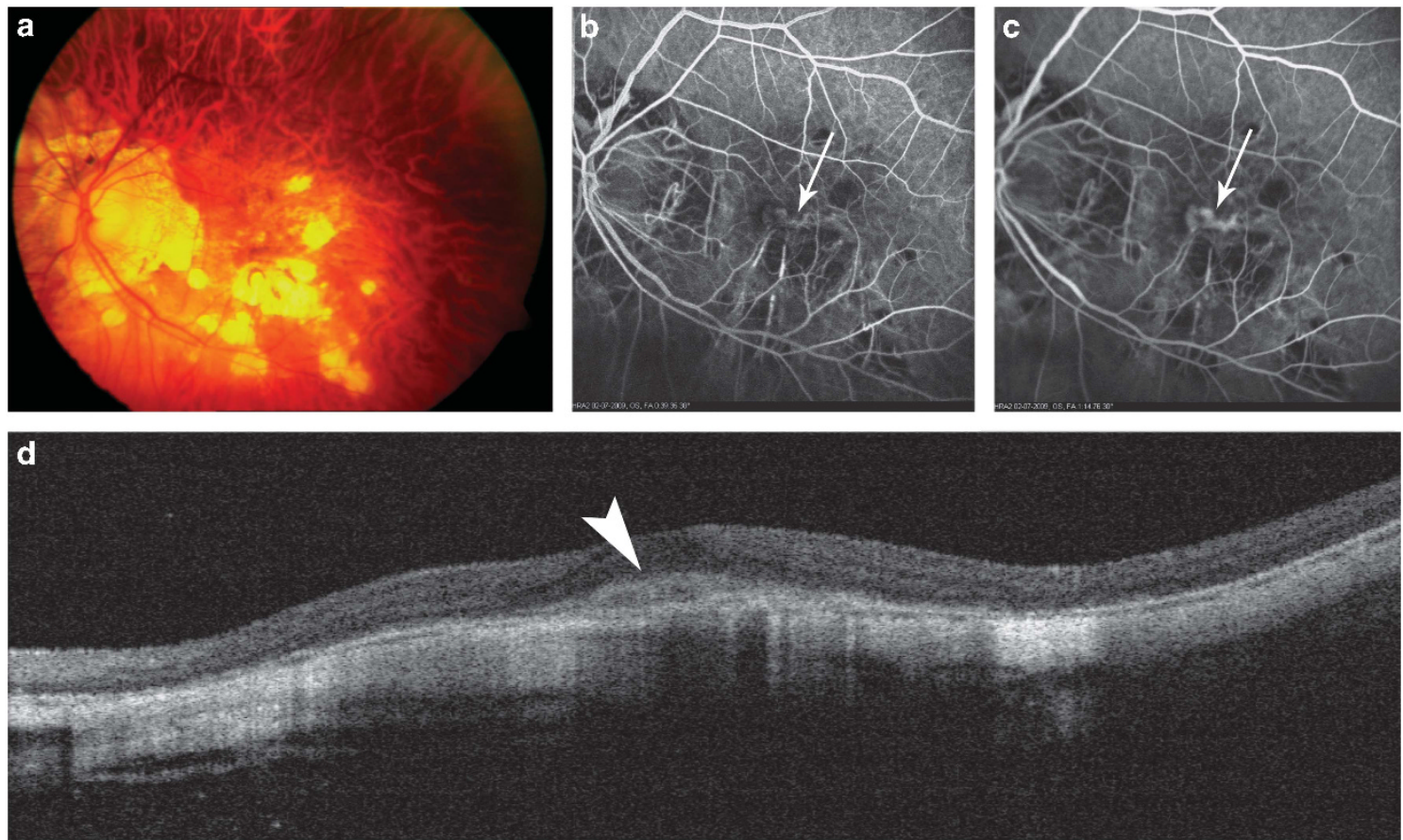

Figure 1 A color fundus photograph (a) shows patches of chorioretinal atrophy in a high-myopic fundus without any subretinal hemorrhage or choroidal neovascular membrane (CNV). Early phase of fluorescein angiography (b) shows early hyperfluorescence (arrow) with diffuse leakage (arrow) in the late phase of fluorescein angiography (c). Fluorescein angiography (b and c) is suspicious of the presence of CNV. However, a spectral domain optical coherence tomography (d) scan shows a hyper-reflective lesion (arrowhead) at the outer retina without any evidence of intraretinal or subretinal fluid.
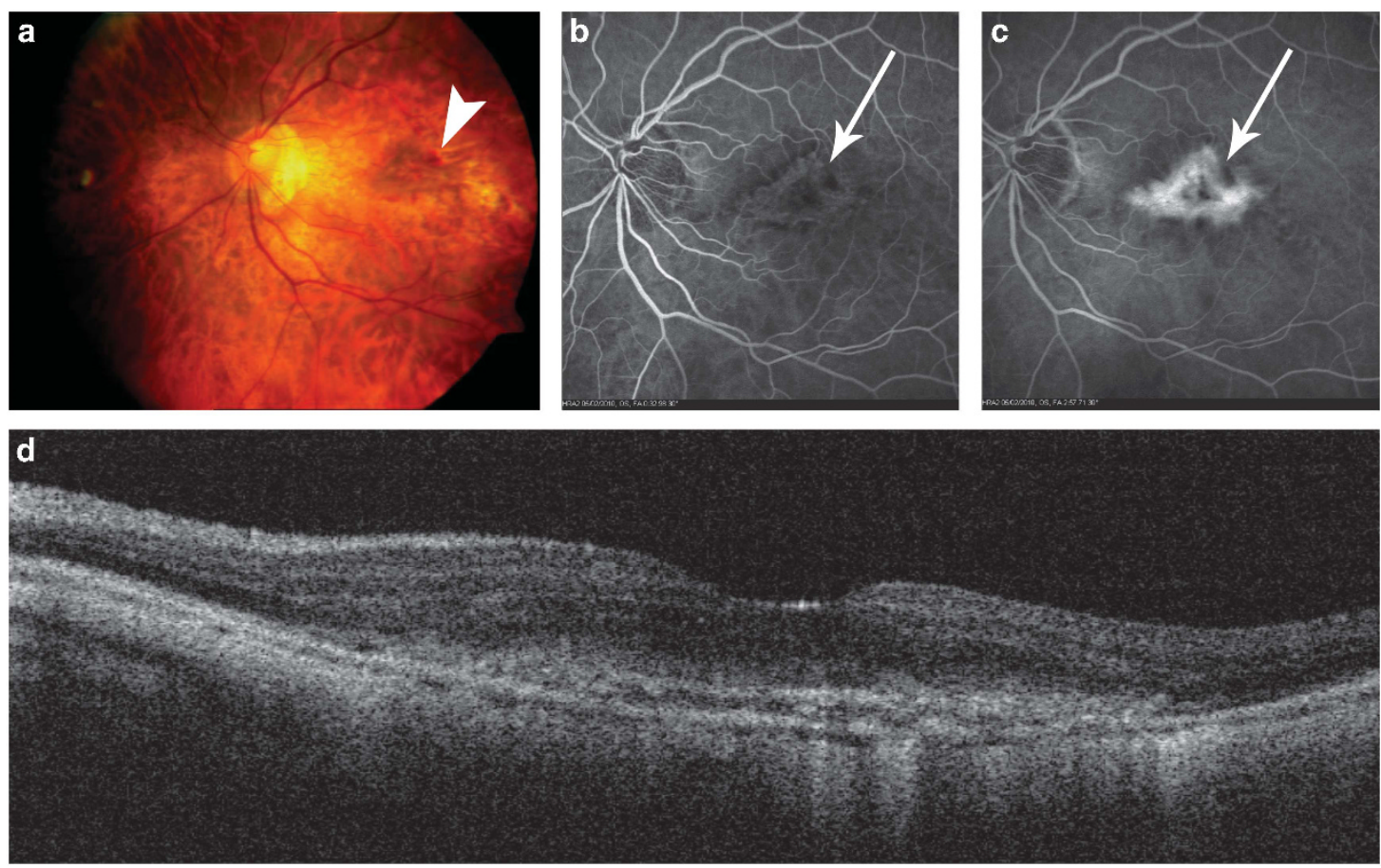

Figure 2 A color fundus photograph (a) shows patches of subretinal hemorrhage (arrowhead) in a high-myopic fundus. Early phase of fluorescein angiography (b) shows early hyperfluorescence (arrow) of the membrane with diffuse hyperfluorescence (arrow) in the late phase of fluorescein angiography (c). Fluorescein angiography (b and c) is suspicious of the presence of a choroidal neovascular membrane. However, a spectral domain optical coherence tomography (d) scan shows a hyper-reflective lesion at the outer retina without any evidence of intraretinal or subretinal fluid. 
When considering a color fundus photo as the gold standard, we found both, FFA and SD-OCT, had 50-60\% sensitivity, with slightly better values for SD-OCT. SD-OCT had slightly better specificity, as well as negative and positive predictive values as shown in Table 1 . The probable reason is that the FFA findings could be confusing due to associated staining and posterior pole changes. In contrast, presence of IRF and/or SRF associated with retinal thickening on SD-OCT almost confirms the diagnosis of CNV. Therefore, we recommend SD-OCT as the preferred test over FFA to rule out the presence of myopic CNV.

Clinicians are always concerned about high-myopic patients who present with recent vision loss, and the clinical examination does not show any obvious CNV lesion. In our study, out of 43 symptomatic eyes, only $53.4 \%$ eyes had clinical diagnosis of $\mathrm{CNV}$, however, a diagnosis of CNV was made on FFA and OCT in 51.1 and $41.8 \%$ eyes, respectively. Therefore, multimodal imaging should be performed to evaluate such symptomatic patients and make a correct diagnosis.

Clinical diagnosis of $\mathrm{CNV}$ in a high myope could be very difficult in absence of subretinal hemorrhage or a membrane. Subretinal hemorrhage could be associated with high myopia, especially the younger age group. There could be the presence of lacquer cracks at the area of hemorrhage. Usually such hemorrhages are very small and disappear in 6-8 weeks ${ }^{12}$ and will not show exudative signs on FFA and SD-OCT. In present study, only one eye had subretinal hemorrhage without a membrane and did not show any leakage on FFA as well. Therefore, it is important to understand that subretinal hemorrhage is not always a sign of $\mathrm{CNV}$ in a high myope.

Our study had inherent limitations of a retrospective study. We did not include indocyanine green angiography information in our study. We did not analyze associated fundus findings in relation with CNV. Strengths of our study include a good sample size and inclusion of patient's presenting complaint and its correlation with clinical findings and information from diagnostic modalities. We had a very strict criterion for image selection; poor image quality or unavailability of any of the images led to exclusion of that eye.

In conclusion, the repeatability and reproducibility for making diagnosis of myopic CNV was better with FFA compared with SD-OCT, however, agreement is very poor between FFA and SD-OCT for making diagnosis of myopic CNV. SD-OCT is comparatively a better tool to rule out the presence of myopic CNV. Multimodal evaluation of patients with acute vision loss without clinical signs of myopic CNV is mandatory for appropriate management.

\section{Summary}

What was known before

- Myopic choroidal neovascualrization is difficult to diagnose on clinical as well as on various imaging modalities. Patient's symptoms may not correlate with the clinical findings and findings on imaging modalities.

What this study adds

- FFA has better repeatability and reproducibility compared with SD-OCT to make the diagnosis of myopic CNV. Agreement between FFA and SD-OCT is poor, however, SD-OCT is a better tool to rule out the presence of myopic CNV.

\section{Conflict of interest}

The authors declare no conflict of interest.

\section{References}

1 Sperduto RD, Seigel D, Roberts J, Rowland M. Prevalence of myopia in the United States. Arch Ophthalmol 1983; 101(3): 405-407.

2 Wang Q, Klein BE, Klein R, Moss SE. Refractive status in the Beaver Dam Eye Study. Invest Ophthalmol Vis Sci 1994; 35(13): 4344-4347.

3 Angle J, Wissmann DA. The epidemiology of myopia. Am J Epidemiol 1980; 111(2): 220-228.

4 Krishnaiah S, Srinivas M, Khanna RC, Rao GN. Prevalence and risk factors for refractive errors in the South Indian adult population: the Andhra Pradesh eye disease study. Clin Ophthalmol 2009; 3: 17-27.

5 Noble KG, Carr RE. Pathologic myopia. Ophthalmology 1982; 89(9): 1099-1100.

6 Ohno-Matsui K, Yoshida T, Futagami S, Yasuzumi K, Shimada N, Kojima A et al. Patchy atrophy and lacquer cracks predispose to the development of choroidal neovascularisation in pathological myopia. $\mathrm{Br} J$ Ophthalmol 2003; 87(5): 570-573.

7 Chan WM, Ohji M, Lai TY, Liu DT, Tano Y, Lam DS. Choroidal neovascularisation in pathological myopia: an update in management. Br J Ophthalmol 2005; 89(11): 1522-1528.

8 Mokwa NF, Ristau T, Keane PA, Kirchhof B, Sadda SR, Liakopoulos S. Grading of age-related macular degeneration: comparison between color fundus photography, fluorescein angiography, and spectral domain optical coherence tomography. J Ophthalmol 2013; 2013: 385915.

9 Milani P, Massacesi A, Setaccioli M, Moschini S, Mantovani E, Ciaccia S et al. Sensitivity of fluorescein angiography alone or with SD-OCT for the diagnosis of myopic choroidal neovascularization. Graefes Arch Clin Exp Ophthalmol 2013; 251(8): 1891-1900.

10 Leveziel N, Caillaux V, Bastuji-Garin S, Zmuda M, Souied EH. Angiographic and optical coherence tomography characteristics of recent myopic choroidal neovascularization. Am J Ophthalmol 2013; 155(5): 913-919. 
11 Keane PA, Liakopoulos S, Chang KT, Heussen FM, Ongchin SC, Walsh AC et al. Comparison of the optical coherence tomographic features of choroidal neovascular membranes in pathological myopia versus age-related macular degeneration, using quantitative subanalysis. Br J Ophthalmol 2008; 92(8): 1081-1085.

12 Soubrane G. Choroidal neovascularization in pathologic myopia: recent developments in diagnosis and treatment. Surv Ophthalmol 2008; 53(2): 121-138. 